\title{
Peripheral Nerve Infection, CTCAE
}

National Cancer Institute

\section{Source}

National Cancer Institute. Peripheral Nerve Infection, CTCAE. NCI Thesaurus. Code C143751.

A disorder characterized by an infectious process involving the peripheral nerves. 